\title{
A Multi-Criteria Portfolio Analysis of Hedge Fund Strategies
}

Complutense

de Análisis

Económico

\section{David E. Allen}

Centre for Applied Financial Studies, University of South Australia, School of Mathematics and Statistics,University of Sydney, and School of Business and Law, Edith Cowan University

\section{Michael McAleer}

Department of Quantitative Finance, College of Technology Management, National Tsing Hua University,Taiwan; Econometric Institute, Erasmus School of Economics, Erasmus University Rotterdam, The Netherlands; Department of Quantitative Economics, Complutense University of Madrid, Spain; Institute of Advanced Sciences,Yokohama National University, Japan

\author{
Abhay K. Singh \\ School of Business and Law, Edith Cowan University
}

\begin{abstract}
This paper features a tri-criteria analysis of Eurekahedge fund data strategy index data. We use nine Eurekahedge equally weighted main strategy indices for the portfolio analysis. The tri-criteria analysis features three objectives: return, risk and dispersion of risk objectives in a Multi-Criteria Optimisation (MCO) portfolio analysis. We vary the MCO return and risk targets and contrast the results with four more standard portfolio optimisation criteria, namely the tangency portfolio (MSR), the most diversi_ed portfolio (MDP), the global minimum variance portfolio (GMW), and portfolios based on minimising expected shortfall (ERC). Backtests of the chosen portfolios for this hedge fund data set indicate that the use of MCO is accompanied by uncertainty about the a priori choice of optimal parameter settings for the decision criteria. The empirical results do not appear to outperform more standard bi-criteria portfolio analyses in the backtests undertaken on our hedge fund index data.
\end{abstract}

Keywords MCO, Portfolio Analysis, Hedge Fund Strategies, Multi-Criteria Optimisation, Genetic Algorithms.

JEL Classification G15, G17, G32, C58, D53.

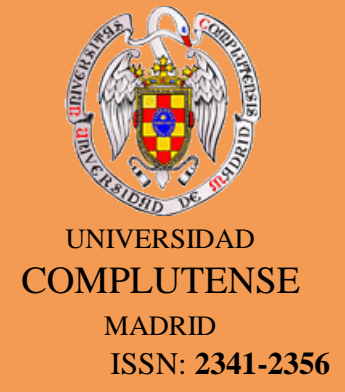

\section{Working Paper no 1703 December, 2016}

WEB DE LA COLECCIÓN: http://www.ucm.es/fundamentos-analisis-economico2/documentos-de-trabajo-del-icaeWorking papers are in draft form and are distributed for discussion. It may not be reproduced without permission of the author/s. 


\title{
A Multi-Criteria Portfolio Analysis of Hedge Fund Strategies
}

\author{
David E. Allen ${ }^{\mathrm{a}}$, Michael McAleer ${ }^{\mathrm{b}}$, and Abhay K. Singh ${ }^{\mathrm{c}}$ \\ ${ }^{a}$ Centre for Applied Financial Studies, University of South Australia, School of Mathematics and Statistics, \\ University of Sydney, and School of Business and Law, Edith Cowan University \\ ${ }^{b}$ Department of Quantitative Finance, College of Technology Management, National Tsing Hua University, \\ Taiwan; Econometric Institute, Erasmus School of Economics, Erasmus University Rotterdam, The Netherlands; \\ Department of Quantitative Economics, Complutense University of Madrid, Spain; Institute of Advanced Sciences, \\ Yokohama National University, Japan \\ ${ }^{c}$ School of Business and Law, Edith Cowan University
}

\begin{abstract}
This paper features a tri-criteria analysis of Eurekahedge fund data strategy index data. We use nine Eurekahedge equally weighted main strategy indices for the portfolio analysis. The tri-criteria analysis features three objectives: return, risk and dispersion of risk objectives in a Multi-Criteria Optimisation (MCO) portfolio analysis. We vary the MCO return and risk targets and contrast the results with four more standard portfolio optimisation criteria, namely the tangency portfolio (MSR), the most diversified portfolio (MDP), the global minimum variance portfolio (GMW), and portfolios based on minimising expected shortfall (ERC). Backtests of the chosen portfolios for this hedge fund data set indicate that the use of MCO is accompanied by uncertainty about the a priori choice of optimal parameter settings for the decision criteria. The empirical results do not appear to outperform more standard bi-criteria portfolio analyses in the backtests undertaken on our hedge fund index data.
\end{abstract}

Keywords: MCO, Portfolio Analysis, Hedge Fund Strategies, Multi-Criteria Optimisation, Genetic Algorithms.

JEL Codes: G15, G17, G32, C58, D53.

\section{Introduction}

A primary purpose of the paper is to examine how expanding a portfolio analysis from bi-criteria, which typically leads to a single criterion being optimal for hedge fund strategies, to multi-criteria, which improves the flexibility in the choice of optimal strategies, but at the possible expense of a single criterion being optimal. This leads to a horses for courses outcome, which requires a wider and more sensible range of strategies to be considered.

Hallerbach and Spronk (2002) reviewed the benefits of incorporating the techniques of multiple criteria analysis into financial decision making, in general, both at the level of the firm and in investment decisions. The focus in this paper is on investment decisions, namely the portfolio selection decision. In our analysis we contrast standard mean-variance bi-criteria portfolio selection, where the efficient set is a frontier with multi-criteria portfolio analysis and the efficient frontier becomes an efficient surface.

While it is not yet standard for there to be additional criteria in portfolio selection, there has been recent growing discussion of the topic in the literature (see, for example, Steuer et al., (2005), Steuer et al. (2015), Qi et al. (2015), and Utz et al. (2015)). Deb (2001, 2011) and Deb et

Email address: michael.mcaleer@gmail.com 
al. (2011) have considered the use of evolutionary algorithms in multi-objective optimization and demonstrated applications in both product design and manufacturing, and in portfolio optimisation. Pfaff (2016) has recently promoted the effectiveness of various packages available in the open-source statistical software R library, as a pathway to the effective use of tri-criteria portfolio optimisation as a financial decision tool. In the empirical analysis undertaken in this paper, we follow the lead and use the suggested capabilities of the various libraries available in $\mathrm{R}$.

Wallenius et al. (2008) reviewed the various areas of multiple-criteria decision making and multi-attribute utility theory. They noted that evolutionary multi-objective optimization (EMO) has emerged as a new field with strong ties to multi-criteria decision making. The application of evolutionary algorithms starts with an initial population, and updates the population by using processes designed to mimic natural survival-of-the-fittest principles and genetic variation operators to improve the average population from generation to generation in a stochastic manner. The goal is to converge to a diverse final population of points that represents the nondominated set. This approach is adopted in the paper and applied to a financial portfolio optimisation problem.

Markowitz (1952) developed portfolio theory as a bi-criteria model in the context of his meanvariance model. The mean refers to efforts to maximise the expected return of the stochastic variable, which constitutes the porfolio's return, while the variance, which is Markowitz's risk proxy, reflects the endeavour to minimize the variance of the stochastic portfolio return. Hence, the Markowitz portfolio selection criteria, which has remained the predominant model for the past six decades, is a bi-criteria model reflecting attempts to maximise the expected return of the portfolio, while simultaneously minimising its variance.

There have been various attempts to modify Markowitz's portfolio selection model to combat its perceived weaknesses. Allen et al. (2016) mention some of the problems attached to the issue of estimation risk, and note that Markowitz (1959, p. 206) suggests that: "Problems concerning the proper information to serve as the basic inputs concerning securities are outside the scope of this monograph. There are no magic formulas to supplant the sources of information and the rules of judgement of the security analyst". Historical data are often used to estimate the required means and covariances, but this leads to estimation risk which, in turn, can lead to extreme and unstable portfolio weights over time. Michaud (1989, p. 3) suggests that: "The traditional MV [mean-variance] procedure often leads to financially irrelevant or false 'optimal' portfolios and asset allocations. In fact, equal weighting can be shown to be superior to MV optimization in some cases", and "MV optimizers are, in a fundamental sense, estimation-error maximizers".

One approach to this problem involves the application of Bayesian techniques to adjust for estimation risk. Some of the original suggested adjustments were either based on the use of diffuse priors (see, for example, Barry (1974) and Bawa et al. (1979)), or 'shrinkage' estimators. The latter were explored by Jobson et al. (1979), Jobson and Korkie (1980) and Jorion (1985).

Roy (1952) developed his 'safety-first' asset selection criteria, Markowitz $(1952,1959)$ considered a number of downside risk measures as an alternative to mean-variance analysis, and Rockafellar et al. (2006, 2007a, 2007b) developed the mean-deviation approach to portfolios as an extension to the classic mean-variance approach. They generalised the results to the one fund theorem, CAPM, plus the derivation of market equilibrium for investors using different deviation measures. More recently, Zabarankin et al. (2014) have extended the CAPM with draw-down measures of betas and alphas. 
An evaluation of the relative effectiveness of naive diversification relative to standard Markowitz portfolio optimisation is provided by DeMiguel et al. (2009). A further contrast with the efficacy of down-side risk measures and dynamic optimisation strategies is provided by Allen et al. (2016). The concern in the current paper is not with these issues, but with the merits of adding further decision criteria to the basic Markowitz optimisation.

The paper is organised into five sections: the introduction is followed by a discussion of research methods in Section 2, which discusses tri-criteria portfolio optimisation strategies in contrast to variants of the Markowitz bi-criteria optimisation. Both general approaches are adopted in this paper, beginning with tri-criteria MCO analysis, which is contrasted with various bi-criteria optimisation approaches, namely the tangency portfolio (MSR), the most diversified portfolio (MDP), the global minimum variance portfolio (GMW), and portfolios based on minimising expected shortfall (ERC). Section 3 introduces the dataset, its characteristics, and research methods, while Section 4 presents the empirical results, including an analysis of various back-tests. A conclusion follows in Section 5.

\section{Models}

Qi et al. (2015) suggest that a multi-criteria opimization criterion can be written as:

$$
\begin{gathered}
Z_{i}=f_{1}(x) \\
\cdot \\
\cdot \\
\cdot \\
\max \left\{Z_{k}=f_{k}(x)\right\} \\
\text { s.t. } x \in S,
\end{gathered}
$$

where $k$ is the number of objectives, and $S \subset R_{n}$ is the feasible region in decision space. As (1) has more than one objective, there is another version of the feasible region, $Z \subset R_{k}$ in criterion space, where $Z=\left\{z \mid z_{i}=f_{i}(x), x \in S\right\}$, with reference to which $z=\left(z_{1}, \ldots . . z_{k}\right)$, is a criterion vector. In criterion space, $\bar{z} \in Z$ is not dominated if there does not exist an $x \in S$ such that $f_{i}(x) \geq f_{i}(\bar{x})$ for all $i$, with at least one strict inequality. Otherwise, $\bar{z}$ is dominated. The set of all nondominated criterion vectors is called the nondominated set, and is designated $N$. In decision space, $x \bar{\in} S$ is efficient if its criterion vector $z=\left(f_{1}(\bar{x})^{-}, \ldots, f_{k}(\bar{x})\right.$ is nondominated. Otherwise, $\bar{x}$ is inefficient. The set of all efficient points is called the efficient set, and is designated $E$. In the form above, the purpose of (1) is to compute all of $N$ and $E$ for use by the decision maker.

In Markowitz (1952), the bi-criterion format is:

$$
\begin{gathered}
\min \left\{z_{1}=x^{T} \sum x\right\} \text { variance, } \\
\max \left\{z_{2}=\mu^{T} x\right\} \text { expectedreturn, } \\
\text { s.t. } x \in S
\end{gathered}
$$

where $x \in R^{N}, N$ is the number of securities considered, the $x_{i}$ components are the security weights, $\sum$ is the covariance matrix, and $\mu$ is the vector of individual security expected returns. 


\subsection{A tri-criteria model}

An additional objective can be added to (2) to form a tri-criterion model:

$$
\begin{gathered}
\min \left\{z_{1}=x^{T} \sum x\right\}, \\
\max \left\{z_{2}=\mu^{T} x\right\}, \\
\max \left\{z_{3}=\ell^{T} x\right\}, \\
\text { s.t. } \ell^{T} x=1 .
\end{gathered}
$$

Steuer et al. (2007) suggest that there are many candidates for a third criterion, but in the analysis which follows we will use diversification with respect to risk contributions of assets. Deb et al. (2011) discuss practical problems encountered in the implementation of portfolio analysis, suggesting that standard QP solvers face difficulties in the presence of discontinuities and other complexities. They remark that genetic algorithms may be better placed to deal with these types of issues.

The approach using evolutionary algorithms (EA) was discussed earlier by Streickert et al. (2003). EAs are population based stochastic optimization heuristics inspired by Darwin's Theory of Evolution. An EA searches through a solution space in parallel by evaluating a set (population) of possible solutions (individuals). An individual gives a solution by representing the decision variables $w_{i}$, in our context the choice of portfolio weights. An EA starts with a random initial population $P_{0}$. Then the 'fitness' of each individual is determined by evaluating the relevant objective function. After the best individuals $P_{t}^{\prime}$ are selected, new individuals for the next generation $P_{t+1}$ are created from $P_{t}^{\prime}$. New individuals are generated by altering the individuals of $P_{t}^{\prime}$ through random mutation and by mixing the decision variables of multiple parents through crossover. Then the generational cycle repeats itself until a breaking criteria is fulfilled, as shown in Figure 1, which is taken from Streikert et al. (2003). This basic approach is shown in the right-hand section of Figure 1, labelled Fig. 1. EA scheme. 


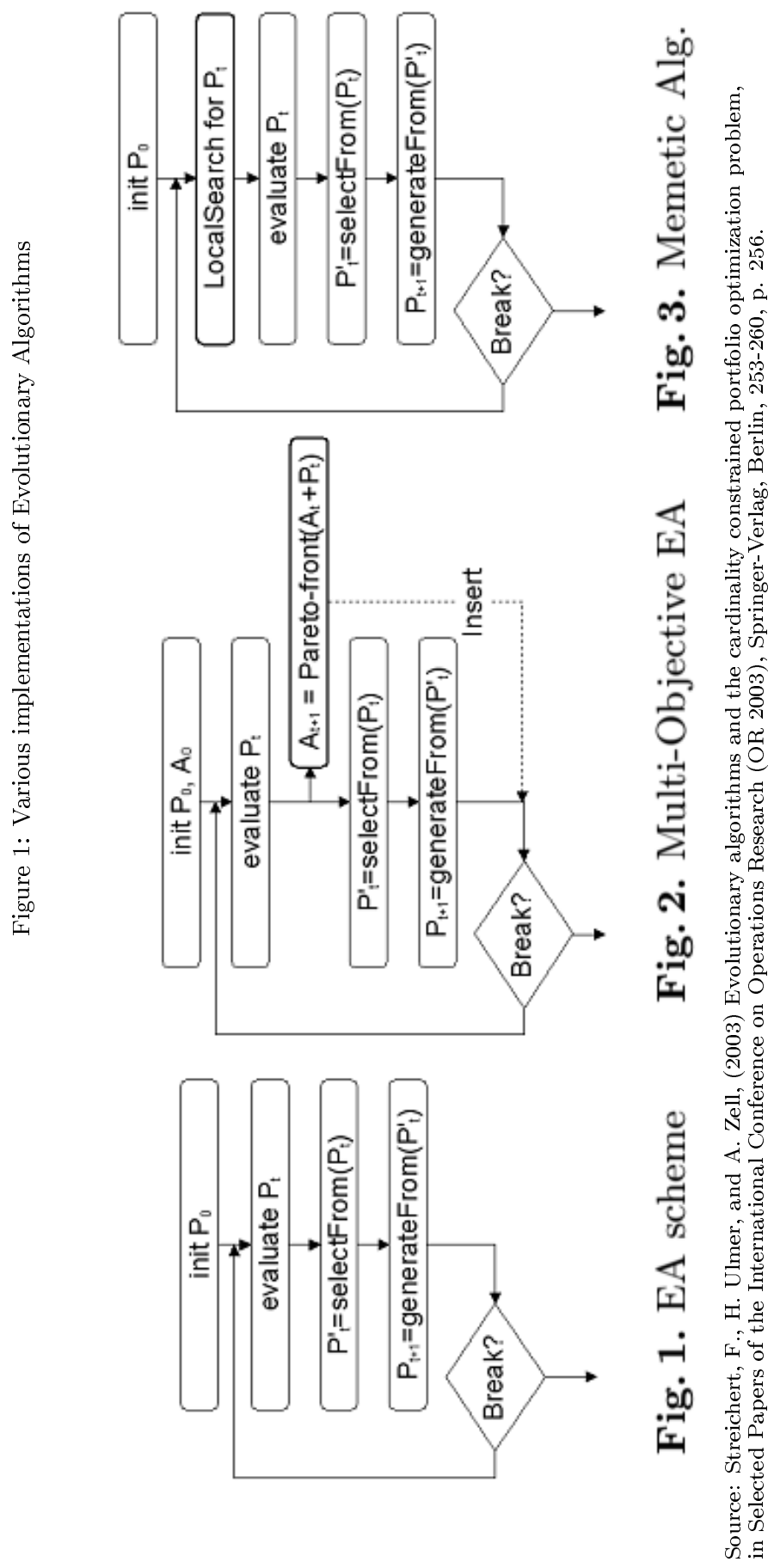


A multi-objective EA scheme is shown in the middle section of Figure 1, labelled 'Fig. 2. MultiObjective EA'. Given the population-based search strategy and the simple selection strategy, EAs can be readily extended to multi-objective optimization problems and then, by using selection based on multiple objective values like the Pareto-dominance criteria, by adding an archive population, $A_{t}$, used to maintain the currently known Pareto-front.

The process of multi-objective optimization permits two goals to be reached. On the one hand, the solutions should be as close to the global Pareto-optimal front as possible and, on the other, the solutions should also cover the whole Pareto front. The first goal is often achieved through elitism by replacing random individuals in $P_{t}$ with individuals on the Pareto front $A_{t}$, as shown in the middle diagram of Figure 1, labelled 'Multi-Objective EA'. The second goal can be achieved by punishing individuals who are too close together (Fitness Sharing).

Memetic Algorithms (MA) serve to extend EA by adding an arbitrary (possibly problem specific) local search heuristic before evaluating the population $P_{t}$, as shown in the third section of Figure 1 labelled 'Fig. 3. Memetic Alg'.

\section{Data and Research Methods}

\subsection{Data}

The data consist of hedge fund strategy index data taken from EurekaHedge ${ }^{1}$. We use the equally weighted monthly series, in which the monthly index values are the respective mathematical means (average) of the monthly returns of all hedge fund constituents in the index at that time. Unlike other indices, they are not asset weighted, or median returns. The returns reported in the database, as well as being included and calculated for indices, are monthly returns provided by hedge funds on a monthly basis. The returns are measured in terms of the gain/loss of the total portfolio values by performance (net of all fees).

The equally-weighted return for each hedge fund strategy Return $_{S}$ is calculated as:

$$
\operatorname{Return}_{S}=\sum_{i=1}^{n}\left(\frac{\operatorname{Return}_{i}}{n}\right)
$$

where $n$ is the number of funds included, and $\operatorname{Return}_{i}$ is the return on an individual fund. The indices simply give an overview of the average performance of hedge funds, without attempting to highlight monthly inflows and overweight the performance of certain funds. Equal weighting also encompasses funds denominated in different currencies, such as US dollar, euro and Japanese yen. The index is purely an average of the performance of the constituent funds in their local currencies.

Only 'unique' funds are selected for the index, with no duplicate share classes, currency denominations, onshore, and offshore versions of the same fund or series. If Eurekahedge discover new funds with historical performance, these funds are immediately included in the index and all returns are rebalanced accordingly. If a fund dies, its track record remains permanently in the

\footnotetext{
${ }^{1}$ (see:http://www.eurekahedge.com/Indices/)
} 
index. Furthermore, since the rationale behind the Eurekahedge suite of indices is relative benchmarking, rather than making them investible, funds that are closed for further capital inflows are also included in an index.

We have adopted these Eureka hedge fund strategy indices for the purpose of our multi-criteria portfolio analysis because hedge fund returns are likely to have non-Gaussian return series with long tails. Our third investment criterion is the dispersion of risk objectives and the prior was that dispersion is a likely characteristic of hedge fund returns. Bali et al. (2013) suggest that hedge funds' extensive use of derivatives, short selling, and leverage and their dynamic trading strategies create significant nonnormalities in their return distributions, and O'Doherty et al. (2016) discuss the problems this leads to in hedge fund performance assessment.

We adopt nine series for our analysis: The Eurekahedge Long Short Equities Hedge Fund Index (Bloomberg Ticker - EHFI252), Eurekahedge Arbitrage Hedge Fund Index (Bloomberg Ticker EHFI285), Eurekahedge CTA/Managed Futures Hedge Fund Index (Bloomberg Ticker - EHFI286), Eurekahedge Distressed Debt Hedge Fund Index (Bloomberg Ticker - EHFI287), Eurekahedge Event Driven Hedge Fund Index (Bloomberg Ticker - EHFI288), Eurekahedge Fixed Income Hedge Fund Index (Bloomberg Ticker - EHFI289), Eurekahedge Long Short Equities Hedge Fund Index (Bloomberg Ticker - EHFI252), Eurekahedge Macro Hedge Fund Index (Bloomberg Ticker EHFI253), Eurekahedge Multi-Strategy Hedge Fund Index (Bloomberg Ticker - EHFI254), and the Eurekahedge Relative Value Hedge Fund Index (Bloomberg Ticker - EHFI255) (see the appendix for further details). 
Figure 2. Plots of Eurekahedge Fund Strategy Indices Monthly Arithmetic Returns
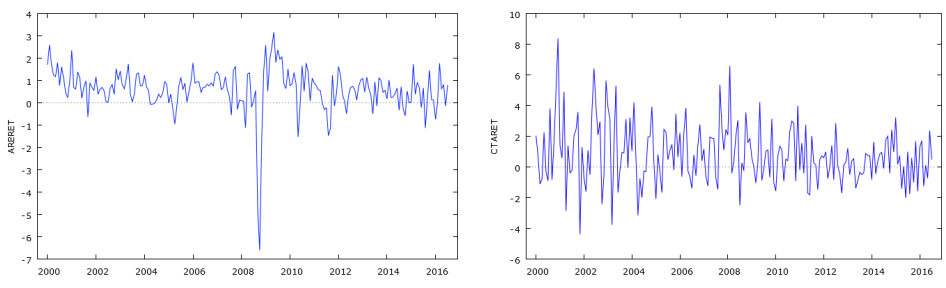

(a) Arbitrage and CTA/Managed Futures
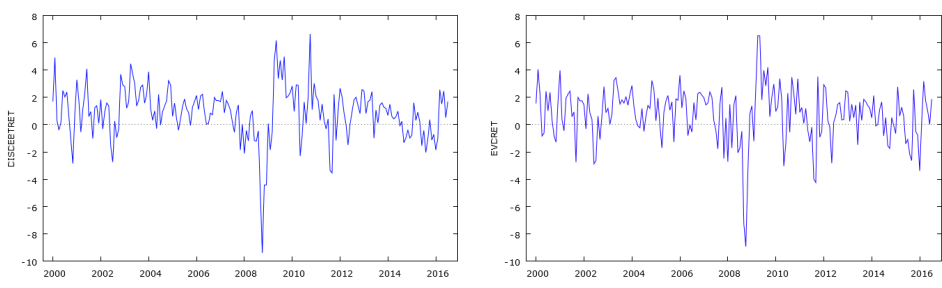

(b) Distressed Debt and Event Driven
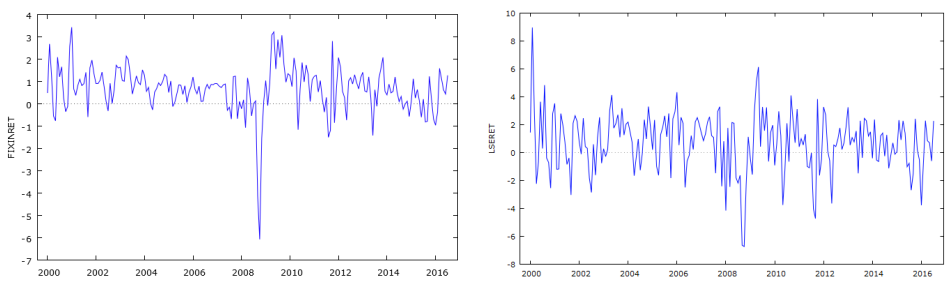

(c) Fixed Interest and Long/Short Equity
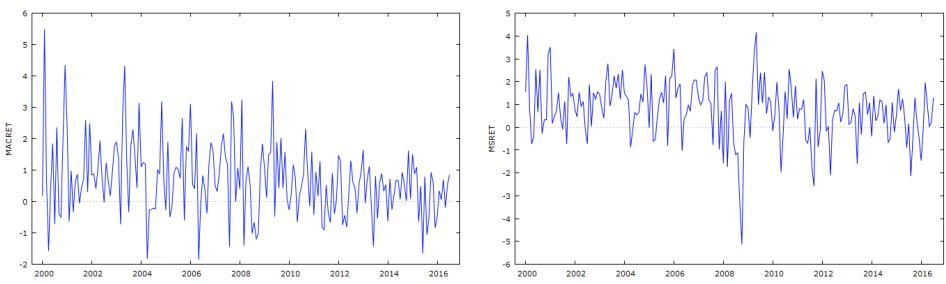

(d) Macro and Multi-Strategy

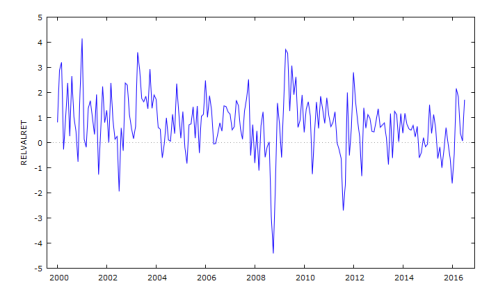

(e) Relative Value 


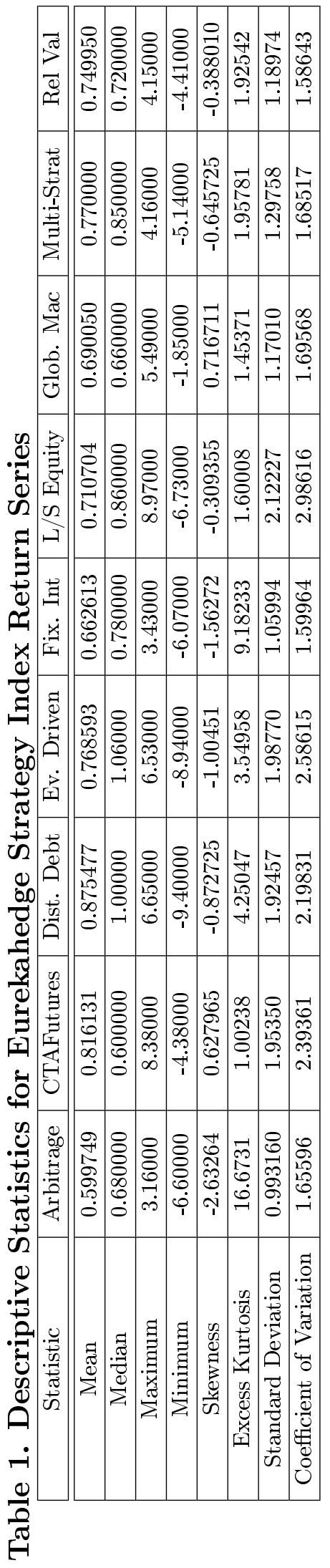


Figure 3. QQ plots of the Eurekahedge Strategy Return Series
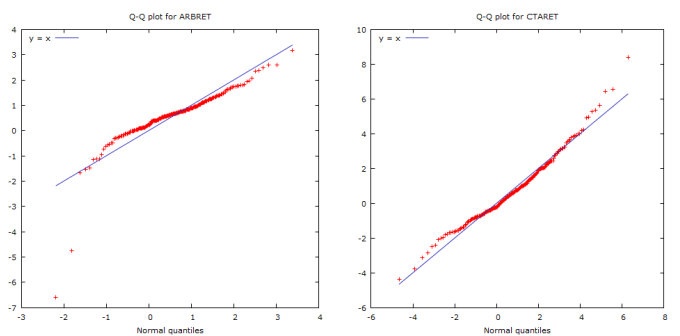

(f) Arbitrage and CTA/Managed Futures
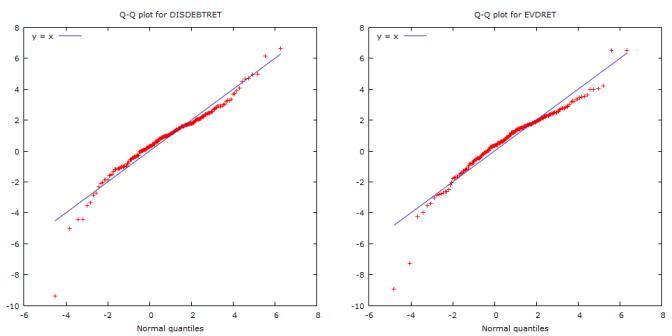

(g) Distressed Debt and Event Driven
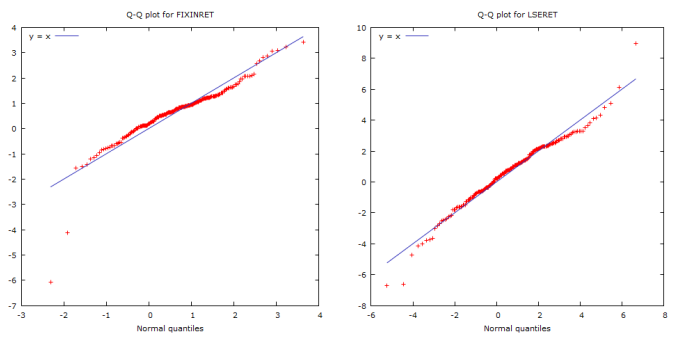

(h) Fixed Interest and Long/Short Equity
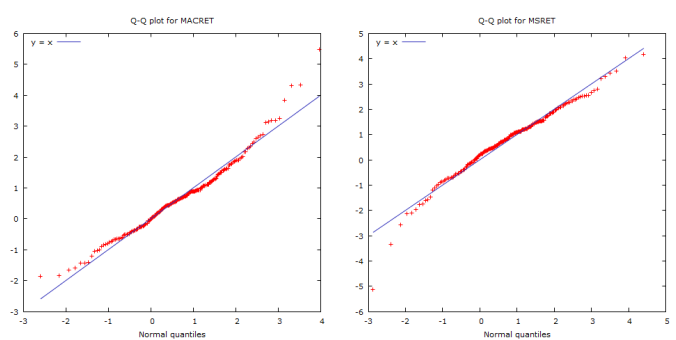

(i) Global Macro and Multi-Strategy

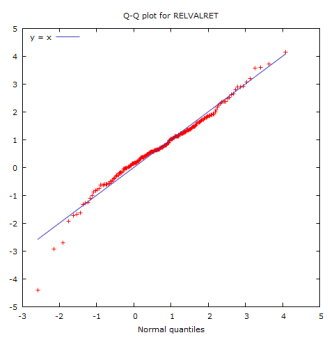

(j) Relative-Value 
These indices should provide a relatively accurate indication of hedge fund strategy returns, and are interesting candidates for a tri-criteria portfolio analysis. However, they are not directly investible, and an investor located in a particular currency domain would be subject to further currency translation effects, as they would need to transform all returns in a common currency from the multi-currency denominated indices. Further description of the composition of the Eureka Hedge Strategy Indices is given in the Appendix.

The time series plots of monthly Eurekahedge Fund Strategy Index returns, shown in Figure 2 , reveal that most hedge fund strategies showed a large fall in returns around the middle of 2008, at the height of the Global Financial Crisis. This was not the case for strategies involving CTA/Managed Futures and Global Macro strategies.

The descriptive statistics for the hedge fund strategy returns, as shown in Table 1, suggest that the mean monthly return varies from around $0.6 \%$ to almost $0.9 \%$ for Distressed Debt, while the maximum can be as high as $8.97 \%$ for Long/Short Equity, or as low as $-9.4 \%$ for Distressed Debt. The standard deviations of the returns vary from just under $1 \%$ to $2.1 \%$, while the skewness is negative for 7 of the 9 series. Arbitrage, Distressed Debt, Event Driven and Fixed Interest are the strategies with the most pronounced excess kurtosis. The standard deviation of the monthly returns varies from $1 \%$ to $2 \%$, while the coefficient of variation varies from a low of around $1.6 \%$, in the case of Arbitrage, to a high of almost 3\%, in the case of Long/Short Equity. This is relevant to our third opimisation criteria in which we seek to minimise the dispersion of risk.

QQ plots of the Eurekahedge Strategy return series are shown in Figure 3. The most striking feature of the QQ plots is the extreme deviation of returns in the lower tail from a Gaussian distribution. The strategies involving Arbitrage, Distressed Debt, Event Driven, Fixed Interest, Long/Short Equity, Multi-Strategy and Relative Value, all deviate sharply below the QQ plot line in their left tails, while, in contrast, Global Macro deviates above the line. Global Macro similarly deviates above the line in its upper tail. This is an issue for portfolio optimisation based on the use of the first two moments. It will be interesting to see if the addition of a third optimisation criterion, based on risk dispersion, changes the empirical results significantly.

\subsection{Research Methods}

In order to undertake the analysis, we use the $\mathrm{R}$ language and environment for statistical computing and graphics. A number of packages are used from the R library, including: Multiple Criteria Optimization Algorithms and Related Functions (MCO), Financial Risk Modelling and Portfolio Optimisation with R (FRAPO), Scatterplot3d, Akima, Fields, Rmetric's package fPortfolio, Econometric tools for performance and risk analysis (PerformanceAnalytics), and Cone Constrained Convex Problems (CCCP). Pfaff (2016) demonstrated that it was possible to combine these libraries in $\mathrm{R}$ to undertake multi-criteria portfolio optimisation. The paper draws on his computer code, and has been modified for the purposes of our analysis.

As was discussed in Section 2.1, we are seeking to achieve nondominated solutions that are consistent with Pareto efficiency. We can obtain positions on the efficient frontier, either by use of the previously discussed genetic algorithms or by means of multi-criteria classical decision optimisation. Our data set of 9 Eurekahedge fund strategy returns will constitute the asset universe to be analysed on the basis of the standard mean return and total covariance risk, together with the third criteria of diversification with respect to risk contributions of assets. 
Pfaff (2016) considers the multi-criteria optimisation in the following terms:

$$
\begin{gathered}
\text { minimize } f_{m}(\omega), \quad m=1,2, \ldots, M \\
\text { s.t. } \quad g_{j}(\omega) \geq 0, \quad j=1,2, \ldots, J \\
h_{k}(\omega)=0, \quad k=1,2, \ldots, K \\
\omega_{i}^{L} \leq \omega_{i}, \leq \omega_{i}^{U}, \quad, i=1,2, \ldots, n .
\end{gathered}
$$

As discussed in Section 2.1, the problem has M (conflicting) objective functions and $\mathrm{n}$ constrained variables (weights). A solution $\omega \hat{\in} \Omega$ is efficient in the Pareto or nondominated sense if there is no $\omega \in \Omega$, so that $f_{k}(\omega) \leq f_{k}(\hat{\omega})$ for $k=1, \ldots, p$, and $f_{i}(\omega)<f_{i}(\hat{\omega})$ for some $i \in\{1, \ldots, k\}$. We use the R package MCO to implement a genetic NSGA-II algorithm to find solutions which lie on the Pareto efficient front, and cover the permissible range. However, the process is not guaranteed to find optimal points that are actually on the frontier.

We use multi-criteria optimisation with three objectives; mean return, volatility, and dispersion of risk contributions. The target mean return is set at $6 \%$, and the targeted volatility at $4 \%$. We subsequently vary the target parameters for returns to $2 \%$ and $4 \%$ to explore how that impacts on the empirical results.

A number of comparisons are made with alternative asset allocation strategies. We use code from the fPortfolio and PortfolioAnalytics packages available on R-Forge, which provide appropriate optimisation routines. For example, the objective to minimize portfolio variance is a quadratic problem of the form:

$$
\underset{\omega}{\operatorname{minimize}} \omega^{\prime} \sum \omega
$$

where $\sum$ is the estimated covariance matrix of asset returns, and $\omega$ is the set of weights.

Choueifaty and Coignard (2008) and Choueifaty et al. (2013) have explored the theoretical and empirical properties of diversified porfolios. Choueifaty et al. (2013) present the mathematical properties of the diversification ratio and most diversified portfolio (MDP), and investigate the optimality of the MDP in a mean-variance framework. The Diversification Ratio (DR) is the ratio of the portfolio's weighted average volatility to its overall volatility. The measure captures the concept of diversification, whereby the volatility of a long-only portfolio is less than or equal to the weighted sum of the asset's volatilities. The DR of a long-only portfolio is greater than or equal to 1 , and equals unity for a single asset portfolio.

They consider a universe of $N$ risky assets $\left\{S_{1}, \ldots . ., S_{N}\right.$, with volatility $\sigma=\sigma_{i}$, correlation matrix $C=\left(\rho_{i}, j\right)$ and covariance matrix $\sum=\left(\rho_{i, j}, \sigma_{i} \sigma_{j}\right)$, with $1 \leq i, j \leq N$. Let $\omega=\left(\omega_{i}\right)$ be the weights of the long only portfolio, $\sigma(\omega)$ its volatility, and $(\omega \mid \sigma)=\sum_{i} \omega_{i} \sigma_{i}$ its average volatility. The diversification ratio $D R(\omega)$ of a portfolio is defined as the ratio of its weighted average volatility and its volatility:

$$
D R(\omega)=\frac{(\omega \mid \sigma)}{\sigma(\omega)}
$$

Choueifaty et al. (2013) develop the properties of the most diversified portfolio (MDP) and 
demonstrate that, in this long-only setting, maximising the DR is equivalent to maximising the Sharpe ratio. For the purposes of comparison, we calculate the MDP and its Sharpe ratio.

The $\mathrm{R}$ package, portfolioAnalytics, has other routines that can be applied in a portfolio meanvariance optimisation framework such as, computing the tangency portfolio on the efficient set, which in our subsequent analysis we will refer to as MSR. We can also compute the global minimum variance portfolio, which we term PMGV.

Boudt et al. (2008) explored the estimation and decomposition of downside risk for portfolios with non-normal returns. The portfolio returns are defined as $r_{p}=\omega^{\prime} \mu$ and the portfolio variance as $m_{2}=\omega^{\prime} \sum \omega$, meaning that $\sum$ is the covariance matrix. Given the assumption of Gaussian distributions, the Value-at-Risk (VaR) and Expected Shortfall (ES) of the portfolio can be computed as:

$$
\begin{gathered}
V a R_{\alpha}=-\omega^{\prime} \mu+\sqrt{m_{2}} \Phi^{-1}(\alpha), \\
E S_{\alpha}=-\omega^{\prime} \mu+\sqrt{m_{2}} \frac{1}{\alpha} \phi\left[\Phi^{-1}(\alpha)\right],
\end{gathered}
$$

where $\phi(\cdot)$ is the density and $\Phi^{-1}(\cdot)$ is the quantile function of the standard normal distribution.

Boudt et al. (2008) demonstrate how to use asymptotic expansions to account for the asymmetry and heavy tails in financial returns to accommodate non-Gaussian distributions, and note that these metrics are included in the $\mathrm{R}$ package performanceAnalytics. We use this metric to calculate riskparity optimisation where ES is the expected shortfall. We compare all multi-criteria optimisation results (MCO) with all these other metrics, and also compare their Sharpe ratios. The empirical results are presented in Section 4.

\section{Empirical Results}

We analyse the nine series of Eurekahedge fund returns using the R package MCO to undertake a trivariate portfolio analysis with a target rate of return of $6 \%$, and a target volatility of $4 \%$, while minimising the dispersion of risk contributions. As we are working in three dimensions, compared with the customary two-dimensional portfolio analysis, the tri-dimensional analysis produces an efficient or Pareto optimal surface, as opposed to an efficient frontier. The map of this surface is shown in Figure 4. 


\section{Figure 4. Surface of Pareto Efficient Solutions}

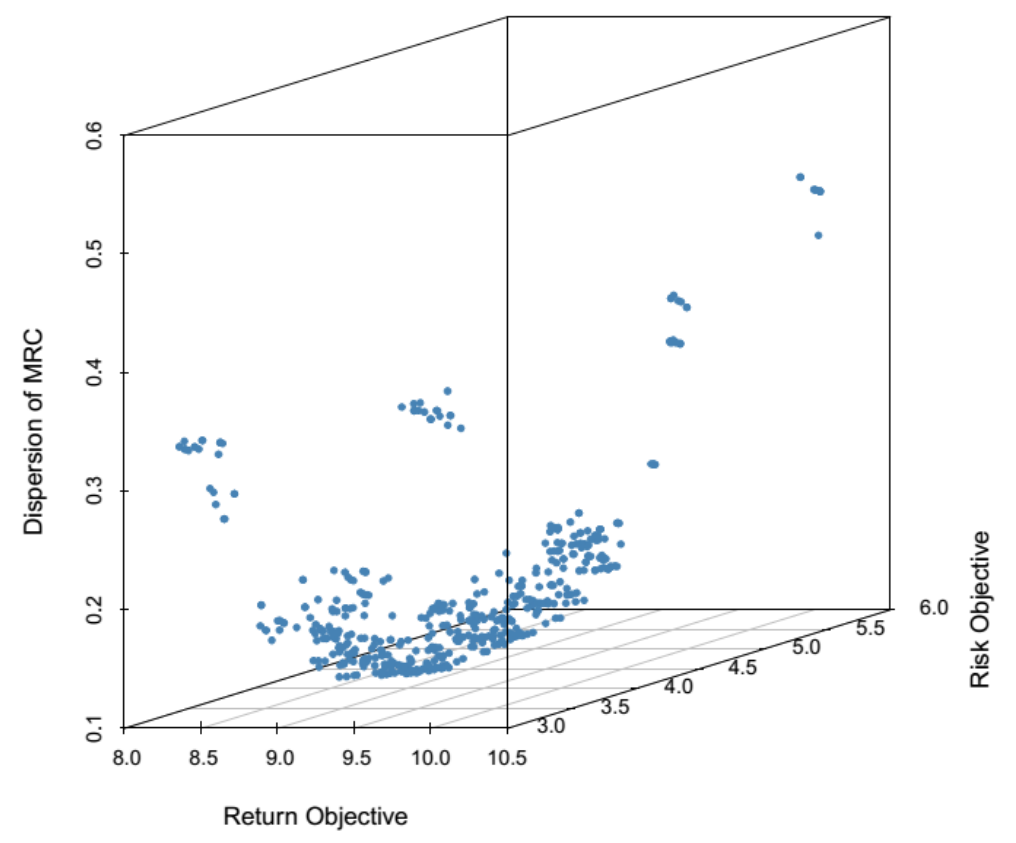

In order to obtain a visual feel for how the genetic algorithm is faring, and to check that the values make intuitive sense, we exported the values of the three criteria "ans" generated by the analysis, and explored their pairwise behaviour. Table 2 provides descriptive statistics for the return, risk, and dispersion of the 500 portfolios generated by the analysis. The mean monthly return of our created portfolios is $1.54 \%$, the mean risk is just under $1 \%$, and the mean dispersion is 0.023 . The coefficient of dispersion is lower for returns at 0.066 , higher for risk with a value of 0.12 , and much higher for dispersion with a value of 0.39 .

Table 2. Summary Statistics of the Return, Risk and
Dispersion of the Generated Portfolios
\begin{tabular}{|c|c|c|c|}
\hline Statistics & Return & Risk & Dispersion \\
\hline \hline Mean & 1.54909 & 0.968101 & 0.233474 \\
\hline Standard Deviation & 0.102686 & 0.124511 & 0.0919030 \\
\hline Minimum & 1.35370 & 0.785550 & 0.117111 \\
\hline Maximum & 1.73356 & 1.24914 & 0.562545 \\
\hline Coefficient of Variation & 0.0662883 & 0.128614 & 0.393632 \\
\hline
\end{tabular}

We then produced some pairwise graphs of the three metrics to check that they are behaving in an intuitively sensible way. The graphs are shown in Figure 5. The results are re-assuring in that, when we combine risk with return in the 500 generated portfolios, we do obtain a positive relationship, 
Figure 5. Pairwise Graphical Analysis of the Joint Behaviour of Return, Risk and Dispersion.
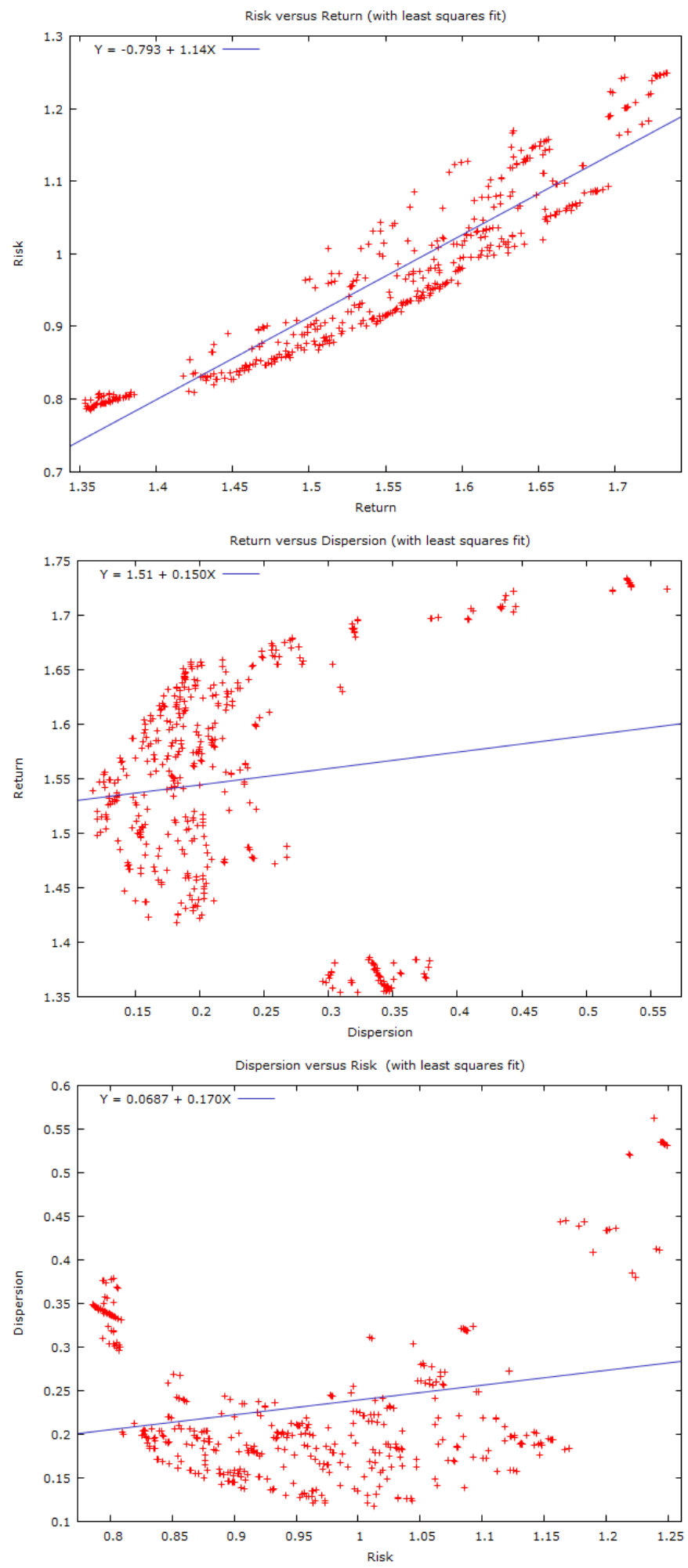
so that we cannot gain a greater return without taking on greater risk. The relationship between return and dispersion, depicted in the middle diagram in Figure 5, shows that if we operate in the top left-hand corner of the diagram, we can generate a greater return by taking on more dispersion.

Finally, the bottom diagram in Figure 5 combines dispersion with risk, and reveals that there are potential gains to be made if we operate in the bottom left-hand quadrant of the diagram. In this segment, we can combine relatively low dispersion with low risk. The segment to be avoided is the top right-hand side of the diagram, where we combine high risk with high dispersion. Hence, the tri-criteria relationships reveal the possibility of benefits from considering the three metrics. The relationships in the lower diagrams in Figure 5 are clearly non-linear, even though the diagram shows a superimposed OLS regression line. This also suits our purposes, as non-linearity increases the potential diversification benefits.

We explore the relationship between the characteristics of these portfolios using the nonparametric Spearman's rank correlation coefficients. The Spearman's rank correlation between return and risk is very high, with rho $=0.959$, which is highly significant. The Spearman's rank correlation coefficient between return and dispersion, has a value of rho $=0.142$, which is also highly significant. However, the Spearman's rank correlation coefficient between risk and dispersion has rho $=-0.013$, which is insignificant.

The effect of combining these effects is shown in Figure 6, which provides an image plot of the efficient set with contour lines superimposed. The efficient portfolios lie in the upper left-hand quadrant of Figure 6, in which area the investor can maximize return while minimizing the

Figure 6. Contour Plot of the Efficient Set

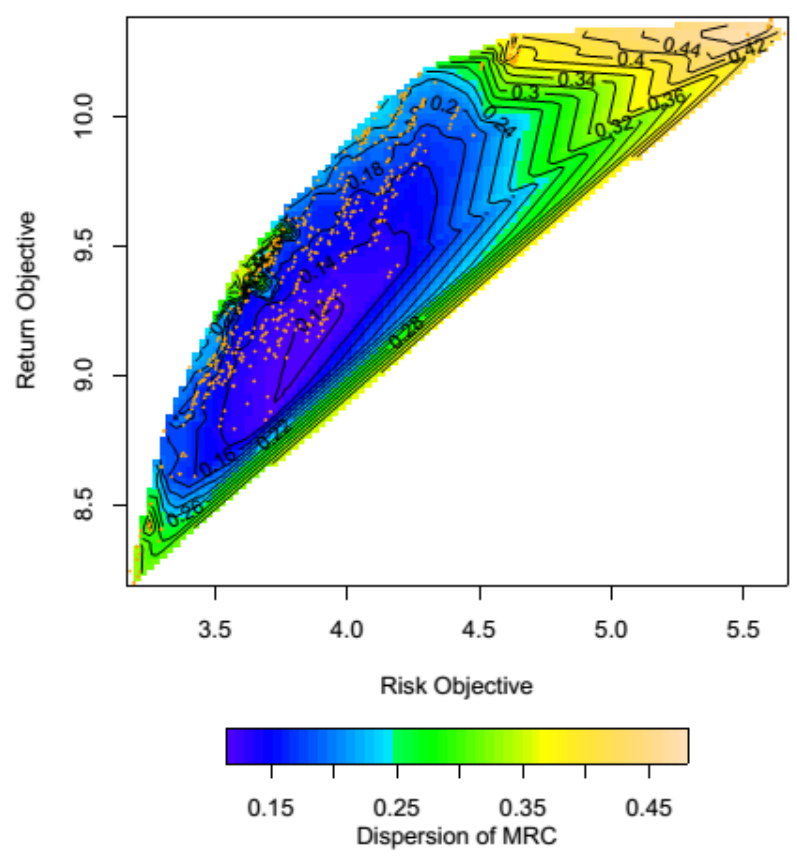

risk and the dispersion of risk. The balance between the three will be determined by the investor's preferences, which we have not yet specified. An indication of the relative attractiveness 
of a multi-criteria investment strategy, as compared with a more standard bi-criteria strategy, will be explored via the Sharpe ratio scores for the various strategies.

A comparison of the portfolio value outcomes in a backtest of the different strategies over time is shown in Figure 7. MCO represents the multi-criteria portfolio which, in this first case, has a target rate of return of $6 \%$ and a target risk of $4 \%$. It can be seen that it produces the highest value in Figure 7 from 2005 to 2010 when it is overtaken by MDP, the Most Diversified Portfolio. The next most valuable strategy in portfolio value terms is ERC, the Expected Short Fall portfolio. MSR represents the tangency portfolio with the efficient frontier, and produces a value that is similar to that of the Global Minimum Variance portfolio, GMW. From the middle of 2010 its moves above GMW.

\section{Figure 7. Backtest Comparison of Allocation Strategies, MCO Return Target 6\% Volatility 4\%}

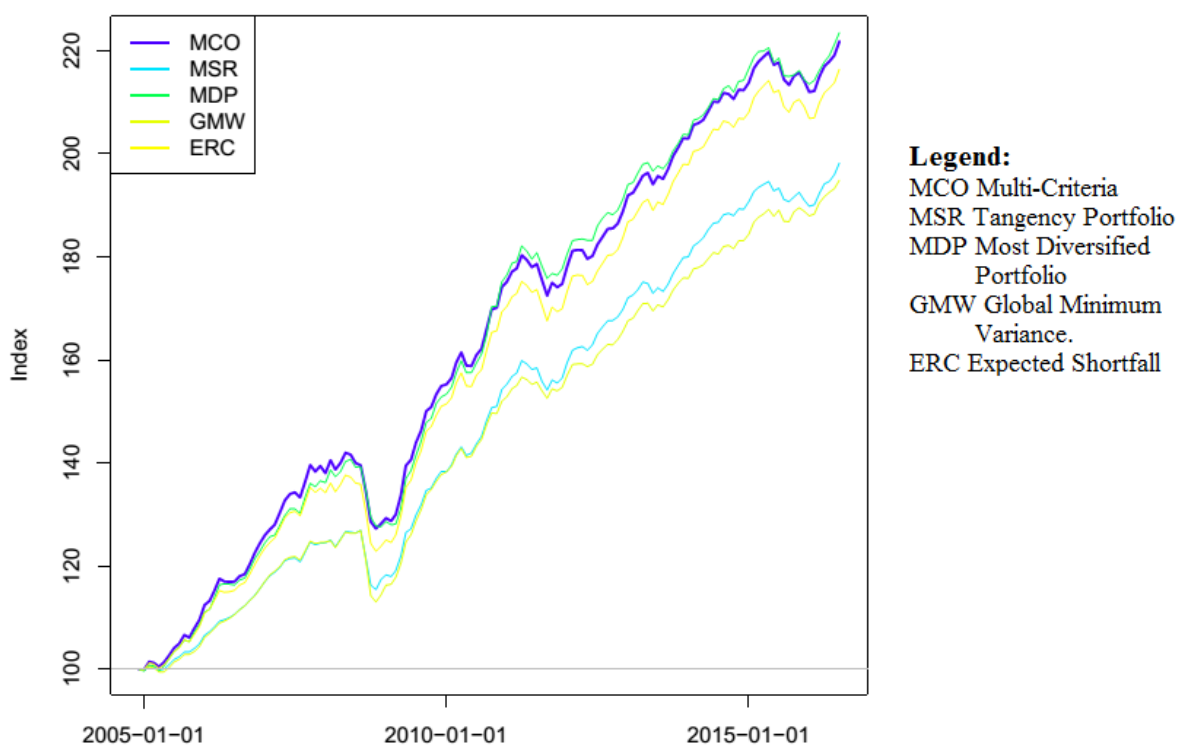

Table 3 summarises the average return, risk, Sharpe Ratios, and Value-at-Risk, VaR, of these portfolio strategies. It confirms that MDP produces the highest average return, in percent per annum (p.a.), followed by MCO, ERC, MSR and GMV. However, the risk is lowest for MSR, at 0.034 in Standard Deviation terms p.a., followed by GMV at 0.035, MDP at 0.039, ERC at 0.40, and MCO at 0.041. MDP has the highest Sharpe Ratio at 1.864 and the lowest VaR at 1.313. MSR has a Sharpe Ratio of 1.768 and VaR of 1.340, followed by MCO with a Sharpe Ratio of 1.744 and VaR of 1.514. ERC has a Sharpe Ratio of 1.710 and VaR of 1.547, while GMW has a Sharpe Ratio of 1.710 and $\mathrm{VaR}$ of 1.488 .

The difficulties in choosing an optimal portfolio strategy are evident in these various outcomes. The MDP approach optimises the Sharpe Ratio, and in this data set with the given parameter settings, produces the highest return, highest Sharpe Ratio, minimum VaR, but only the third lowest standard deviation. The GMW strategy produces the lowest return, the lowest Sharpe ratio, the second lowest standard deviation, and a median VaR. MCO, the multi-criteria approach, 
produces the second highest return, the highest standard deviation, a median Sharpe Ratio, and the second highest VaR.

Table 3. Return Risk Characteristics of Alternative Strategies,
\begin{tabular}{|c|c|c|c|c|c|}
\hline MCO Return Target $6 \%$ Risk 4\% \\
\hline Statistics & MCO & MSR & MDP & GMW & ERC \\
\hline Return (p.a.) & 0.071 & 0.061 & 0.072 & 0.059 & 0.069 \\
\hline StdDev. Risk (p.a.) & 0.041 & 0.034 & 0.039 & 0.035 & 0.040 \\
\hline Sharpe Ratio & 1.744 & 1.768 & 1.864 & 1.688 & 1.710 \\
\hline VaR (p.a.) & 1.514 & 1.340 & 1.313 & 1.488 & 1.547 \\
\hline
\end{tabular}

In order to explore the sensitivity of our results to the target parameter settings, we switched the target rate of return to 2 p.a. and kept the target risk at $4 \%$. In terms of the values of the portfolios in the backtest, as shown in Figure 8, MCO dominates, while the weakest performance in terms of value of the portfolio is given by GMW, the global minimum variance portfolio. The combined metrics shown in Table 4 present a different picture.

\section{Figure 8. Backtest Comparison of Allocation Strategies, Target Return $2 \%$ Risk $4 \%$}

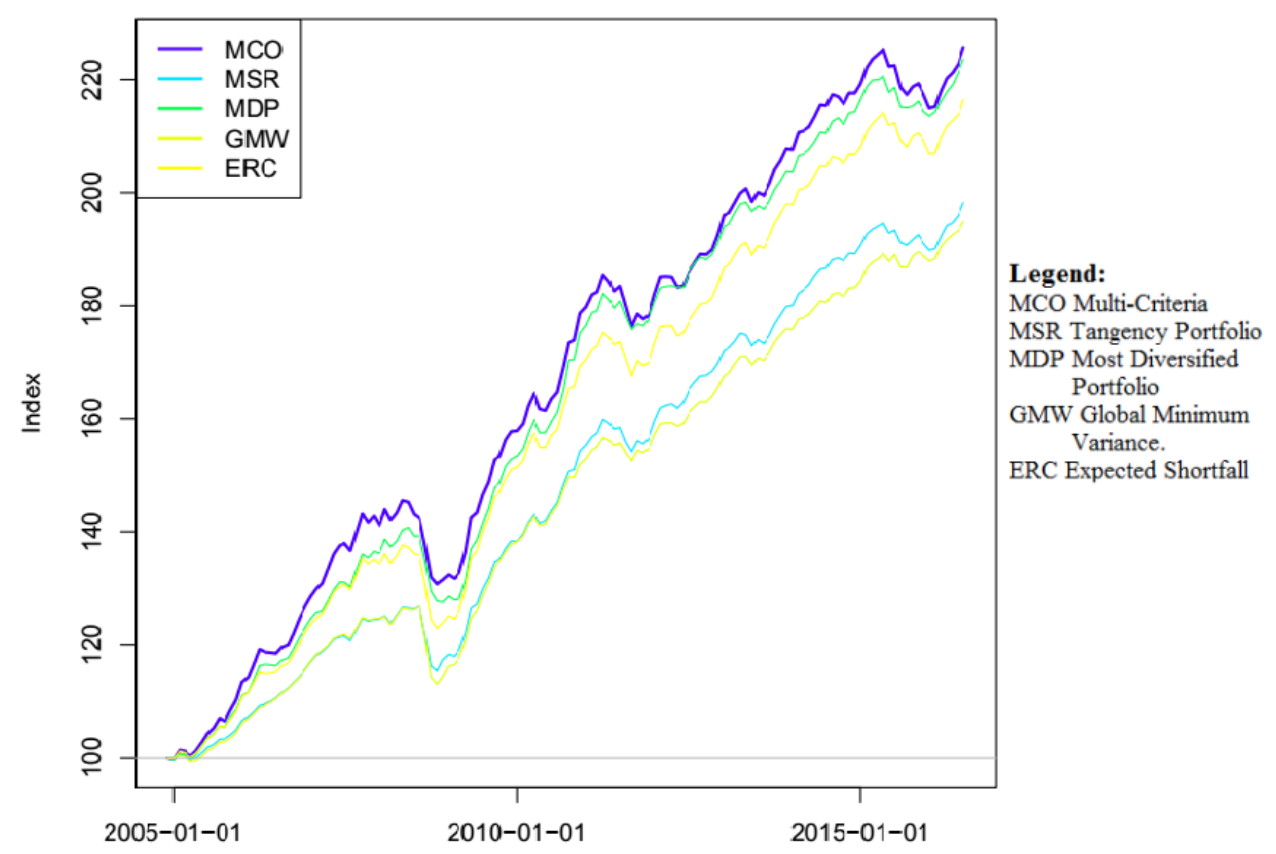

The highest return, as previously mentioned, comes from the MCO strategy, but it also produces the highest standard deviation, the second lowest Sharpe Ratio, and the highest VaR. The MDP approach provides the second highest return, median standard deviation, the highest Sharpe Ratio, and the lowest VaR. Table 4 shows the return and risk characteristics of the portfolios selected under the various strategies when the MCO strategy was a target of $2 \%$ return and $4 \%$ risk. 
Table 4. Return Risk Characteristics of Alternative Strategies, MCO Return Target 2\% Risk $4 \%$

\begin{tabular}{|c|c|c|c|c|c|}
\hline Statistics & MCO & MSR & MDP & GMW & ERC \\
\hline \hline Return (p.a.) & 0.073 & 0.061 & 0.072 & 0.059 & 0.069 \\
\hline StdDev. Risk (p.a.) & 0.043 & 0.034 & 0.039 & 0.035 & 0.040 \\
\hline Sharpe Ratio & 1.693 & 1.786 & 1.864 & 1.688 & 1.710 \\
\hline VaR (p.a.) & 1.565 & 1.340 & 1.313 & 1.488 & 1.547 \\
\hline
\end{tabular}

MCO again dominates the backtest, in terms of returns, with an average return of 0.073 p.a., but MDP is not far behind with 0.072 p.a.. The other metrics, MDP dominates, with a median standard deviation 0.039, highest Sharpe Ratio of 1.864 and lowest VaR of 1.313. In contrast, MCO has the highest standard deviation of 0.043 p.a., a Sharpe Ratio of 1.693 and the highest VaR of 1.565 .

We performed one further set of analyses and set the target for MCO at $4 \%$ risk and $4 \%$ return. The results of the backtest of the values of the portfolio strategies are shown in Figure 9. The MCO strategy again dominates the backtest in terms of the portfolio values, but on the other metrics relating to risk, as shown in Table 5, it does not fare so well. It has the highest standard deviation of 0.042 p.a., median Sharpe Ratio of 1.736, and second highest VaR at 1.508 p.a. .

\section{Figure 9. Backtest Comparison of Allocation Strategies,} Target Return 4\% Risk 4\%

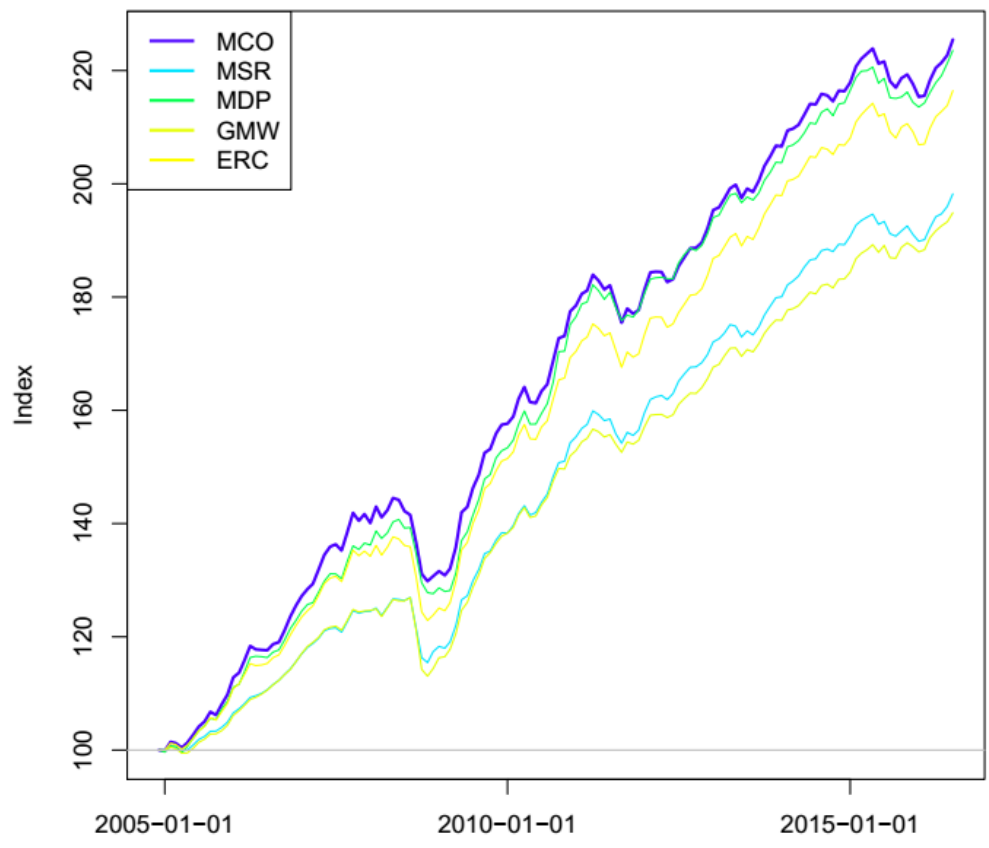

Legend:

MCO Multi-Criteria

MSR Tangency Portfolio

MDP Most Diversified Portfolio

GMW Global Minimum

Variance.

ERC Expected Shortfall 


Table 5. Return Risk Characteristics of Alternative Strategies,
\begin{tabular}{|c|c|c|c|c|c|}
\hline MCO Return Target $4 \%$ Risk $4 \%$ \\
\hline \hline Statistics & MCO & MSR & MDP & GMW & ERC \\
\hline Return (p.a.) & 0.073 & 0.061 & 0.072 & 0.059 & 0.069 \\
\hline StdDev. Risk (p.a.) & 0.042 & 0.034 & 0.039 & 0.035 & 0.040 \\
\hline Sharpe Ratio & 1.736 & 1.786 & 1.864 & 1.688 & 1.710 \\
\hline VaR (p.a.) & 1.508 & 1.340 & 1.313 & 1.488 & 1.547 \\
\hline
\end{tabular}

Once again, MDP has the best relative performance, with a return of $0.072 \%$, standard deviation of 0.039 p.a., which is the median, highest Sharpe Ratio of 1.864, and lowest VaR at 1.313 p.a..

The three sets of results achieved by varying the MCO return and risk targets reveal that it is not easy to implement a multi-criteria portfolio optimisation strategy, in practice. If we set a relatively high target return of $6 \%$, with a target risk of $4 \%$, then Table 3 shows that the backtest indicates an achieved return of $0.071 \%$. Paradoxically, if we lower the target return to $2 \%$, but maintain the target risk at $4 \%$, Table 4 shows that the backtest reveals we achieve a higher return of $0.073 \%$.

However, the target return of $6 \%$ and a risk target of $4 \%$ produces the lowest standard deviation of the three strategies considered, at 0.041 p.a. This strategy also produces the highest Sharpe ratio of the three target settings of 1.744. The lowest VaR of the three MCO strategies considered is produced by using a target of $4 \%$ return and $4 \%$ risk, with a value of 1.508 p.a. in the backtest. However, the highest Sharpe Ratio of 1.744 is produced by the first MCO strategy of a target return of $6 \%$ and target risk of $4 \%$.

Table 6 provides a summary of the empirical findings and their potential contradictions. The highest returns of 0.073 p.a. shown in bold in Table 6 , are provided by an MCO strategy with a

\section{Table 6. Return Risk Characteristics of Alternative Strategies}

\begin{tabular}{|c|c|c|c|c|c|c|c|}
\hline Statistics & $\begin{array}{c}\text { MCO Ret 6\% } \\
\text { Risk 4\% }\end{array}$ & $\begin{array}{c}\text { MCO Ret 2\% } \\
\text { Risk 4\% }\end{array}$ & $\begin{array}{c}\text { MCO Ret 4\% } \\
\text { Risk } 4 \%\end{array}$ & MSR & MDP & GMW & ERC \\
\hline \hline Return (p.a.) & 0.071 & $\mathbf{0 . 0 7 3}$ & $\mathbf{0 . 0 7 3}$ & 0.061 & 0.072 & 0.059 & 0.069 \\
\hline StdDev. Risk (p.a.) & 0.041 & 0.043 & 0.042 & $\mathbf{0 . 0 3 4}$ & $\mathbf{0 . 0 3 9}$ & $\mathbf{0 . 0 3 5}$ & $\mathbf{0 . 0 4 0}$ \\
\hline Sharpe Ratio & 1.744 & 1.693 & 1.736 & 1.786 & $\mathbf{1 . 8 6 4}$ & 1.688 & 1.710 \\
\hline VaR (p.a.) & 1.514 & 1.565 & 1.508 & $\mathbf{1 . 3 4 0}$ & $\mathbf{1 . 3 1 3}$ & $\mathbf{1 . 4 8 8}$ & $\mathbf{1 . 5 4 7}$ \\
\hline
\end{tabular}

lower target return of $2 \%$ and $4 \%$, respectively, than the strategy in the first MCO column, which has a target return of $6 \%$. The three different MCO strategies produce larger standard deviations than all the more customary, bi-criteria strategies. The Sharpe ratios are less conclusive, though MDP with a Sharpe ratio of 1.864 is clearly optimal on this metric. The MCO strategy, though aimed at reducing dispersion, does not produce a low VaR. All the VaRs for the bi-criteria strategy, as shown in bold in Table 6, are lower than the three VaRs produced by the various MCO strategies.

\section{Conclusion}

The paper examined the relationships between five alternative investment strategies. The first, MCO, used multi-criteria portfolio optimisation, while the other four use bi-criteria optimisation, 
namely MSR, MDP, GMW and ERC. The empirical results for this sample of EurekaHedge hedge fund data are inconclusive. One paradox is that setting a lower target return in the MCO analysis, $2 \%$ in this case, with a target risk of $4 \%$, achieves a higher return of $0.073 \%$, as does the MCO analysis with a target return of $4 \%$ and a target risk of $4 \%$, which also achieves $0.073 \%$.

The MDP strategy, which maximises the Sharpe Ratio, does relatively well in terms of this data set, producing the highest Sharpe Ratio of 1.864, as would be expected, but also providing the lowest VaR at 1.313 p.a., and the second highest return at $0.072 \%$. Thus, the MCO criteria have the merit of adding an additional goal to the analysis, but at the cost of complicating the choice of optimal settings.

It is not clear, a priori, what would be the appropriate return and risk targets. Thus, MCO is an interesting extension of portfolio choice, but it is not clear whether it adds much in the way of additional value, while considerably complicating the choice of target settings. Furthermore, the use of genetic algorithms does not guarantee that the suggested solutions will lie on the efficient frontier.

The paper examined, among other issues, how expanding a portfolio analysis from bi-criteria, which typically leads to a single criterion being optimal for hedge fund strategies, to multi-criteria, which improves the flexibility in the choice of optimal strategies, but at the expense of a single criterion being optimal. This important outcome requires a wider and more sensible range of strategies to be considered rather than determining an optimal strategy based on a limited number of possibilities.

An extension of the paper could incorporate dynamic variances and covariances to enable a dynamic analysis of hedge fund strategies, which would give even greater flexibility and optimality in terms bi-criteria and multi-criteria portfolio analysis.

\section{Acknowledgements}

For financial support, the first author acknowledges the Australian Research Council, and the second author is most grateful to the Australian Research Council, National Science Council, Ministry of Science and Technology (MOST), Taiwan, and the Japan Society for the Promotion of Science. 


\section{Appendix}

The Eurekahedge Long Short Equities Hedge Fund Index (Bloomberg Ticker - EHFI252) is an equally weighted index of 1114 constituent funds. The index is designed to provide a broad measure of the performance of underlying hedge fund managers The index is base weighted at 100 at December 1999, does not contain duplicate funds and is denominated in local currencies.

The Eurekahedge Arbitrage Hedge Fund Index (Bloomberg Ticker - EHFI285) is an equally weighted index of 90 constituent funds. The index is designed to provide a broad measure of the performance of underlying hedge fund managers who invest with an arbitrage strategy. The index is base weighted at 100 at December 1999, does not contain duplicate funds and is denominated in local currencies.

The Eurekahedge CTA/Managed Futures Hedge Fund Index (Bloomberg Ticker - EHFI286) is an equally weighted index of 503 constituent funds. The index is designed to provide a broad measure of the performance of underlying hedge fund managers who invest with a cta/managed futures strategy. The index is base weighted at 100 at December 1999, does not contain duplicate funds and is denominated in local currencies.

The Eurekahedge Distressed Debt Hedge Fund Index (Bloomberg Ticker - EHFI287) is an equally weighted index of 29 constituent funds. The index is designed to provide a broad measure of the performance of underlying hedge fund managers who invest with a distressed debt strategy. The index is base weighted at 100 at December 1999, does not contain duplicate funds and is denominated in local currencies.

The Eurekahedge Event Driven Hedge Fund Index (Bloomberg Ticker - EHFI288) is an equally weighted index of 124 constituent funds. The index is designed to provide a broad measure of the performance of underlying hedge fund managers who invest with an event driven strategy. The index is base weighted at 100 at December 1999, does not contain duplicate funds and is denominated in local currencies.

The Eurekahedge Fixed Income Hedge Fund Index (Bloomberg Ticker - EHFI289) is an equally weighted index of 337 constituent funds. The index is designed to provide a broad measure of the performance of underlying hedge fund managers who invest with a fixed income strategy. The index is base weighted at 100 at December 1999, does not contain duplicate funds and is denominated in local currencies.

The Eurekahedge Long Short Equities Hedge Fund Index (Bloomberg Ticker - EHFI252) is an equally weighted index of 1114 constituent funds. The index is designed to provide a broad measure of the performance of underlying hedge fund managers who invest with a long short equities strategy. The index is base weighted at 100 at December 1999, does not contain duplicate funds and is denominated in local currencies.

The Eurekahedge Macro Hedge Fund Index (Bloomberg Ticker - EHFI253) is an equally weighted index of 238 constituent funds. The index is designed to provide a broad measure of the performance of underlying hedge fund managers who invest with a macro strategy. The index is base weighted at 100 at December 1999, does not contain duplicate funds and is denominated in local currencies.

The Eurekahedge Multi-Strategy Hedge Fund Index (Bloomberg Ticker - EHFI254) is an equally weighted index of 250 constituent funds. The index is designed to provide a broad measure of the performance of underlying hedge fund managers who invest with a multi-strategy strategy. The index is base weighted at 100 at December 1999, does not contain duplicate funds and is denominated in local currencies.

The Eurekahedge Relative Value Hedge Fund Index (Bloomberg Ticker - EHFI255) is an equally weighted index of 74 constituent funds. The index is designed to provide a broad measure of the performance of underlying hedge fund managers who invest with a relative value strategy. The index is base weighted at 100 at December 1999, does not contain duplicate funds and is denominated in local currencies. 


\section{References}

[1] Allen, D.E., M. McAleer, R, Powell, and A.K. Singh (2016), Down-side risk metrics as portfolio diversification strategies across the global financial crisis, Journal of Risk and Financial Management, 9(2), 6; doi:10.3390/jrfm9020006

[2] Bali, T.G., S.J. Brown., and K.O. Dermitas (2013), Do hedge funds outperform stocks and bonds?, Management Science, 59 (8), 1887-1903.

[3] Barry, C.B. (1974), Portfolio analysis under uncertain means, variances, and covariances, Journal of Finance 29, 515-522.

[4] Bawa, V. S., Brown, S., and R. Klein (1979), Estimation Risk and Optimal Portfolio Choice, North Holland, Amsterdam,

[5] Boudt, K., B. Peterson, and C. Croux (2008), Estimation and decomposition of downside risk for portfolios with non-normal returns, Journal of Risk, 11,2, 79-103.

[6] Choueifaty,Y., and Y. Coignard (2008), Toward maximum diversification, Journal of Portfolio Management, 34 (4), 40-51.

[7] Choueifaty,Y., T. Froidure, and J. Reynier (2013), Properties of the most diversified portfolio, Journal of Investment Strategies, 2, 1-22.

[8] Deb, K. (2001), Multi-Objective Optimization using Evolutionary Algorithms, UK, Wiley.

[9] Deb, K. (2011), Multi-objective evolutionary optimisation for product design and manufacturing, Chapter in Multi-Objective Optimization Using Evolutionary Algorithms: An Introduction, New York, Springer, pp. 3-34.

[10] Deb, K., R. Steuer, R. Tewari, and R. Tewari (2011), On the effectiveness of a nsga-ii local search approach customized for portfolio optimization, KanGAL Report 2011007, Indian Institute of Technology Kanpur, Kanpur, India.

[11] DeMiguel, V., L. Garlappi, and R. Uppal (2009), Optimal versus naive diversification: how inefficient is the $1 / \mathrm{N}$ portfolio diversification strategy?, Review of Financial Studies, 22, 1915-1953.

[12] O'Doherty, M.S., N.E. Savin, and A. Tiwari (2016) Evaluating hedge funds with pooled benchmarks, Management Science, 62 (1), 69-89.

[13] Hallerbach, W.G. and J. Spronk (2002), The relevance of MCDM for financial decisions, Journal of Multi-Criteria Decision Analysis, 11(4-5), 187-195.

[14] Jobson, J.D., R. Korkie, and V. Ratti (1979), Improved estimation for Markowitz portfolios using James-Stein type estimators, Proceedings of the American Statistical Association, 41, pp. 279-292.

[15] Jobson, J.D., and R. Korkie (1980), Estimation for Markowitz efficient portfolios, Journal of the American Statistical Association, 75, 544-554.

[16] Jorion, P. (1985), International portfolio diversification with estimation risk, Journal of Business, 58, 259-278.

[17] Markowitz, H.M. (1952), Portfolio selection, Journal of Finance, 7(1), 77-91.

[18] Markowitz, H.M. (1959), Portfolio Selection Efficient Diversification of Investments; Cowles Foundation, Wiley, New York. 
[19] Michaud, R.O. (1989), The Markowitz optimization enigma: Is 'optimized' optimal? Financial Analysts Journal, 45, 31-42.

[20] Pfaff, B. (2016), R/Finance 2016: Applied Finance with R, May 2016, Chicago, IL, USA, Talk: Portfolio Selection with Multiple Criteria, available at: http://www.pfaffikus.de/rif.html

[21] Qi, Y., R.E. Steuer, and M. Wimmer (2015), An analytical derivation of the efficient surface in portfolio selection with three criteria, Annals of Operations Research, doi:10.1007/s10479-015-1900-y

[22] Rockafellar, R.T., S. Uryasev, and M. Zabarankin (2006), Master funds in portfolio analysis with general deviation measures, Journal of Banking and Finance, 30, $743-776$.

[23] Rockafellar, R.T., S. Uryasev, and M. Zabarankin (2007a), Optimality conditions in portfolio analysis with general deviation measures, Mathematical Programming, 108, $515-540$.

[24] Rockafellar, R.T., S. Uryasev, and M. Zabarankin (2007b), Equilibrium with investors using a diversity of deviation measures, Journal of Banking and Finance, 31, $3251-3268$.

[25] Roy, A.D. (1952), Safety first and the holding of assets, Econometrica, 3, 431-449.

[26] Streichert, F., H. Ulmer, and A. Zell (2003), Evolutionary algorithms and the cardinality constrained portfolio optimization problem, in Selected Papers of the International Conference on Operations Research, Springer-Verlag, Berlin, pp. 253-260.

[27] Steuer, R., Y. Qi, and M. Hirschberger (2005), Multiple objectives in portfolio selection, Journal of Financial Decision Making, 1DOI: 10.1007/s10479-015-1900-y.

[28] Steuer, R., Y. Qi, and M. Hirschberger (2007), Suitable-portfolio investors, nondominated frontier sensitivity, and the effect of multiple objectives on standard portfolio selection, Annals of Operations Research, 152(1), 297-317.

[29] Steuer, R., M. Wimmer, and M. Hirschberger (2013), Overviewing the transition of Markowitz bi-criterion portfolio selection to tri-criterion portfolio selection, Journal of Business Economics, 83(1), 61-85.

[30] Utz, S., M. Wimmer, and R. Steuer (2015), Tri-criterion modeling for constructing more-sustainable mutual funds, European Journal of Operational Research, 246(1), $331-338$.

[31] Wallenius, J., J.S. Dyer, P.C. Fishburn, R.E. Steur, S. Zints, and K. Deb (2008), Multiple criteria decison making, multiattribute utility theory: Recent accomplishments and what lies ahead, Management Science, 54, 1336-1349.

[32] Zabarankin, M., K. Pavlikov, S. Uryasev (2014), Capital asset pricing model (CAPM) with draw-down measure, European Journal of Operational Research, 234, 508-517. 